\title{
The Preparation of ZnO/AC Composite Photocatalytic Material for Coking Wastewater Treatment
}

\author{
Yan-Fen WANG ${ }^{1, a,{ }^{*}}$, Xiu-Qian DAI ${ }^{2, b}$, Chu-Yang XU ${ }^{1, c}$, Meng-Ting $\mathrm{LI}^{1, \mathrm{~d}}$ \\ ${ }^{1}$ School of Materials Science and Engineering, Anhui University of Science \& Technology, Huainan \\ 232001, Anhui Province, P.R. China \\ ${ }^{2}$ School of Energy and Security, Anhui University of Science \& Technology, Huainan 232001, Anhui \\ Province, P.R. China \\ awangyanfenyu@163.com, ㄷxaust@163.com, 'rchnie@aust.edu.cn, ${ }^{d}$ limengting1717@163.com \\ ${ }^{*}$ Corresponding author
}

Keywords: $\mathrm{ZnO} / \mathrm{AC}$, Coking wastewater, Photocatalytic material.

\begin{abstract}
A simple and low-temperature method was utilized to attach nanostructured $\mathrm{ZnO}$ to the surface of porous activated carbon (AC). The phase structure and surface morphology of the as-prepared sample were characterized. XRD analysis confirmed patterns for the wurtzite hexagonal phase of $\mathrm{ZnO}$ and $\mathrm{AC}$ from the sample. SEM demonstrated that the porous character and relatively larger surface area of $\mathrm{ZnO} / \mathrm{AC}$ composite. In the photocatalytic degradation of coking wastewater, the $\mathrm{ZnO} / \mathrm{AC}$ composite material showed effective photocatalytic activity. It could be attributed to highly efficient capture and transportation of photo-generated electrons from $\mathrm{AC}$ to $\mathrm{ZnO}$.
\end{abstract}

\section{Introduction}

In recent years, nanostructured $\mathrm{ZnO}$ materials have attracted much attention due to their excellent qptical properties and facile synthesis methods, which can be applied in photovoltaic device applications. However, the wide band-gap energy of $\mathrm{ZnO}$ makes the photo-generated electron-hole pairs recombine fast [1]. In addition, its suspention system can easily generate catalyst condensation and is difficult to recycle, which will cause the waste of raw materials. So far, many methods have been used to efficiently generate electron-hole pairs through visible-light excitation, such as ion doping, noble metal loading and incorporation of electron-accepting materials.

As one of cheap carbon materials, activated carbon (AC) not only has great specific surface area and low cost, but also possesses large catalyst load and strong adsorption properties, which has become one of the first targets for photocatalyst carrier [2-4]. Thus, we report an easy one-step low-temperature chemical method to obtain a novel $\mathrm{ZnO} / \mathrm{AC}$ composite photocatalytic material. At the same time, the photocatalytic efficiency of $\mathrm{ZnO} / \mathrm{AC}$ composite photocatalytic material was characterized by the practical photocatalytic degradation effect of tail water from coking wastewater.

\section{Experimental}

The procedures were followed to fabricate $\mathrm{ZnO} / \mathrm{AC}$ composite. First, $\mathrm{NaOH}(10 \mathrm{mmol})$ and $\mathrm{ZnCl}_{2}$ $(0.2726 \mathrm{~g})$ were adding into $50 \mathrm{~mL}$ of a suspension with activated carbon $(50 \mathrm{wt} \%)$. Then, the mixing solution was stirred for $0.5 \mathrm{~h}$ and kept at $80^{\circ} \mathrm{C}$ for $12 \mathrm{~h}$ in condition of seal. Finally, the resulting precipitate was separated with centrifuge, washed thoroughly with deionized water and dried at $60{ }^{\circ} \mathrm{C}$ for $4 \mathrm{~h}$. The obtained sample was denoted as $\mathrm{ZnO} / \mathrm{AC}$.

The X-ray diffraction (XRD) were carried out on a Japan Rigaku D/max- $\chi \mathrm{A}$ X-ray diffractometer equipped with graphite monochromatized high-intensity $\mathrm{Cu}_{\mathrm{K} \alpha}$ source $(\lambda=1.54178 \AA)$. The scanning electron microscopy (SEM) was performed on JEOL JSM-6700F. The UV-vis diffuse absorption spectra was performed on a DUV-3700 DUV-vis-NIR recording spectrophotometer of Shimadzu Corporation. 


\section{Results and Discussion}

XRD patterns of the as-prepared $\mathrm{AC}, \mathrm{ZnO}$ and $\mathrm{ZnO} / \mathrm{AC}$ samples were shown in Fig.1. There are two different kinds of diffraction peaks in $\mathrm{ZnO} / \mathrm{AC}$ composite(Fig. 1b). The two broad peaks centered at $\sim 25.21$ and $\sim 43.51$ were indexed to the (002) planes and (100) planes of graphite in AC (Fig. 1c), respectively. The other diffraction peaks could be assigned to the hexagonal wurtzite structured $\mathrm{ZnO}$ (JCPDS card, 36-1451) in Fig. 1a. These results indicated that the obtained samples belong to a mixture of two phase containing hexagonal $\mathrm{ZnO}$ and graphite.

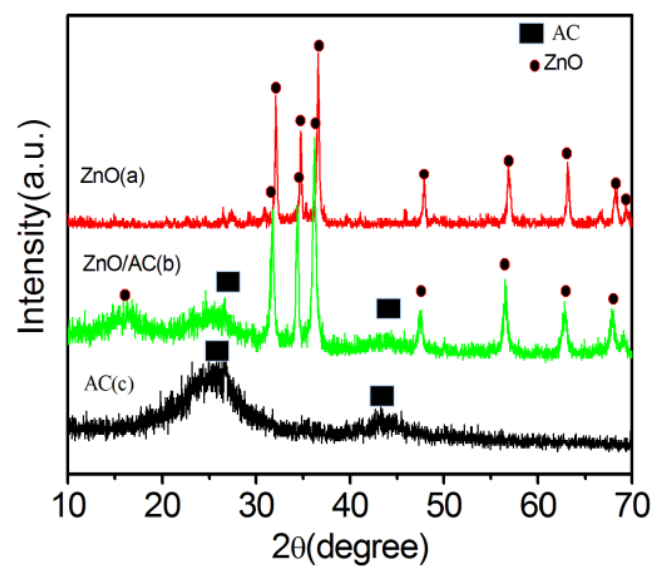

Fig. 1. XRD pattern of the as-prepared $\mathrm{AC}, \mathrm{ZnO}$ and $\mathrm{ZnO} / \mathrm{AC}$ samples

SEM studies provide useful information through the surface morphology of AC and $\mathrm{ZnO} / \mathrm{AC}$ composite. As shown in Fig.2a, it was clearly revealed that multi-layer flake shapes of AC, which can provided larger surface for $\mathrm{ZnO}$ nanoparticles attached onto them. Fig. $2 \mathrm{~b}$ depicted surface texture and porosity nature of $\mathrm{ZnO} / \mathrm{AC}$ composite. $\mathrm{ZnO}$ nanoparticles were uniformly distributed over inside and outside surface of activated carbon. The immobilization of $\mathrm{ZnO}$ in the activated carbon revealed that $\mathrm{ZnO} / \mathrm{AC}$ composites possess the porous character and relatively larger surface area, which will benefit capture and quickly transportation of photo-generated electrons.
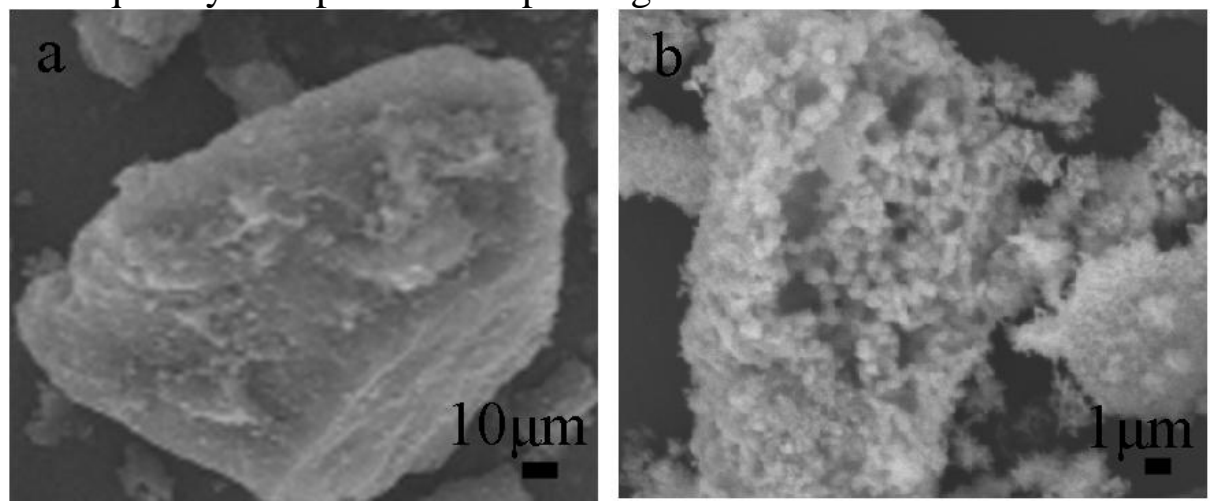

Fig.2. SEM images of the $\mathrm{AC}$ and $\mathrm{ZnO} / \mathrm{AC}$ composite

To evaluate the photocatalytic activity of the as-prepared samples, the photodegradation of practical tail water from coking wastewater, a typical pollutant in the industry, was investigated under the UV-light from Xe lamp irradiation. Fig. 3 shows the UV-Vis absorption spectra of coking wastewater exposure to the light irradiation for various durations in the presence of $\mathrm{ZnO} / \mathrm{AC}$ as photocatalyst, respectively. The characteristic absorption of coking wastewater at $229 \mathrm{~nm}$ decreased rapidly with extension of the exposure time. After irradiation of $120 \mathrm{~min}$, the characteristic absorption of coking wastewater at $229 \mathrm{~nm}$ decreased of about $17 \%$. Though the photocatalytic result is unconspicuous, it indicated that the $\mathrm{ZnO} / \mathrm{AC}$ composite has potential application prospect in the practical coking wastewater treatment. 


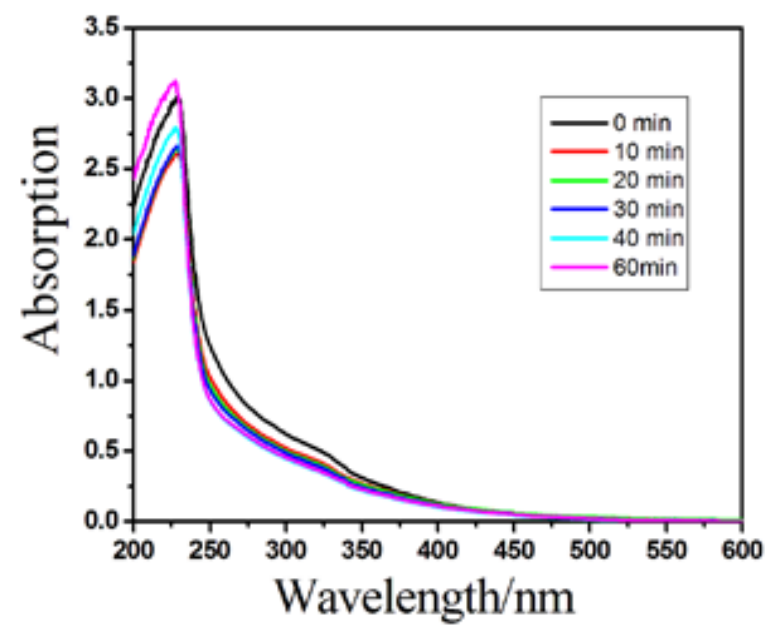

Fig.3.The UV-visible absorption spectra of the as-obtained $\mathrm{ZnO} / \mathrm{AC}$ composite

\section{Conclusions}

A novel $\mathrm{ZnO} / \mathrm{AC}$ composite is prepared by a facile one-step solution-based method under the low temperature. The obtained $\mathrm{ZnO} / \mathrm{AC}$ composite is composed of activated carbon immobilized by $\mathrm{ZnO}$ nanoparticles. For the photodegradation of tail water under the UV-light irradiation, the $\mathrm{ZnO} / \mathrm{AC}$ composite shows potential application prospect in the practical coking wastewater treatment.

\section{Acknowledgments}

This work was financially supported by the Science Research Foundation for Young Teachers of Anhui University of Science and Technology (No. QN201403).

\section{References}

[1] G.H. Chen, Y. Wang, Q.H. Shen, Y.J. Song, G.L. Chen, H. Yang. Synthesis and enhanced photocatalytic activity of 3D flowerlike $\mathrm{ZnO}$ microstructures on activated carbon fiber, Materials Lett. 123 (2014) 145-148.

[2] P. Raizada, P. Singh, A. Kumar, G. Sharma, B. Pare, S.B. Jonnalagadda, Pankaj Thakur. Solar photocatalytic activity of nano- $\mathrm{ZnO}$ supported on activatedcarbon or brick grain particles: Role of adsorption in dye degradation. Applied Catalysis A: General, 486 (2014) 159-169.

[3] P. Muthirulan, M. Meenakshisundararam, N. Kannan. Beneficial role of ZnO photocatalyst supported with porous activated carbon for the mineralization of alizarin cyanin green dye in aqueous solution, J. Adv. Research, 4 (2013) 479-484.

[4] Y.F. Wang, B.X. Li, T.X. Liu, C.Y. Xu, Z.W. Ge. Controllable fabrication of superhydrophobic $\mathrm{TiO}_{2}$ coating with improved transparency and thermostability, Colloids and Surfaces A: Physicochem. Eng. Aspects, 51 (2014) 298-305. 